\title{
STRUCTURAL CHARACTERISTICS OF METAL NANOPARTICLES IN GLASS UPON IRRADIATION-ASSISTED PROCESSING
}

\author{
H. HOFMEISTER ${ }^{\mathrm{a}, *}$, M. DUBIEL ${ }^{\mathrm{b}}$, H. GRAENER ${ }^{\mathrm{b}}$ and J.-C. PIVIN ${ }^{\mathrm{c}}$ \\ ${ }^{\mathrm{a}}$ Max Planck Institute of Microstructure Physics, Weinberg 2, D-06120 Halle, Germany; ${ }^{\mathrm{b}}$ Physics \\ Department, Martin Luther University, D-06108 Halle, Germany; ${ }^{\mathrm{c} C e n t r e ~ o f ~ N u c l e a r ~}$ \\ Spectrometry and Mass Spectrometry, F-91405 Orsay Campus, France
}

Irradiation-assisted processing, i.e. ion, electron and laser irradiation, have been applied to fabricate metal/glass nanocomposites. The particle configurations are studied by transmission electron microscopy to get some insight into the rather complex formation mechanisms. Special attention is given to spheroidally shaped particles surrounded by smaller secondary particles observed upon ion beam mixing of silica/silver/silica layer compounds as well as irradiating femtosecond laser pulses on sodium silicate glass containing spherical silver particles. Another unique type of structure are cavities observed in silver particles formed by high fluence ion implantation into silica as well as upon laser pulse irradiation of silver particles in glass. The experimental findings are discussed in terms of irradiation-induced defect formation and radiation-enhanced diffusion.

Keywords: Metal nanoparticles; Glass; Irradiation

\section{INTRODUCTION}

Metal nanoparticle doped glasses have promising properties for potential application in optical devices $[1,2]$. These properties sensitively depend on their size, shape, density and spatial distribution $[3,4]$. Conventional routes of synthesis, e.g. ion exchange plus thermal processing $[5,6]$, have limited flexibility to control concentration, size and configuration of particles embedded in glass. Recently a number of irradiation-assisted processing techniques have been developed to broaden the range of experimental feasibility. These include impact of ion beams or high-energy irradiation applied to induce, promote or modify particle formation inside the glass. Depending on dose and energy of ions or radiation, different mechanisms are effective. Ion implantation causes metal doping and particle formation [7,8]. Ion irradiation enables redistribution of pre-formed particles [9] and mixing of insulator-metal layer compounds to get particulate composites $[10,11]$. Ionising radiation like X-ray or electron beams cause reduction of metal ions contained in glass $[12,13]$. Laser irradiation may induce redox reactions by local heating [14], or cause various morphological changes by utilising the localised spectral absorption of pre-existing particles $[4,15]$.

\footnotetext{
* Corresponding author.
} 
The specific structures generated by irradiation-assisted processing often are not discernible by diffraction or spectroscopic techniques. Therefore, transmission electron microscopy (TEM) is used to obtain structural information, in particular of highly inhomogeneous configurations. This knowledge is essential to get insight into the rather complex formation mechanisms and to control the properties of such nanocomposite materials. Here we report on ion, laser, and electron irradiation applied to various glasses and discuss the obtained structures in terms of radiation effects of varying type.

\section{EXPERIMENTAL}

Electron irradiation was applied to commercial sodium silicate glass doped with $\mathrm{Ag}$ by an ion exchange procedure whereupon a concentration of about $4.8 \mathrm{Ag}^{+}$ions $/ \mathrm{nm}^{3}$ was achieved. Irradiation processing was performed in a conventional TEM (EM $10 \mathrm{CR}$ ) using an extraordinary large beam current density of $6.4 \mathrm{~A} / \mathrm{cm}^{2}$ at $100 \mathrm{kV}$ acceleration voltage $[12,16]$. Before this processing, the glass was cut into $30 \mathrm{~nm}$ thick slides using an ultramicrotome to allow uniform interaction with the electron beam.

Laser irradiation was applied to commercial sodium silicate glass of the above type, also doped with $\mathrm{Ag}$ by ion exchange. A central region of this glass with nearly monodisperse spherical Ag particles of about $25 \mathrm{~nm}$ size formed by annealing at $600{ }^{\circ} \mathrm{C}$ was chosen for irradiation. To this purpose, linear polarised single laser pulses of $150 \mathrm{fs}$ duration and typically $100 \mu \mathrm{J}$ pulse energy were applied [15]. The wavelength of the laser was fixed around $400 \mathrm{~nm}$ to fit to the surface plasmon resonance of $\mathrm{Ag}$ particles.

Ion irradiation was applied to thin film sandwich structures of $8 \mathrm{~nm} \mathrm{Ag}$ buried within $\mathrm{SiO}_{2}$ layers of $100 \mathrm{~nm}$ thickness deposited on Si substrates using electron beam evaporation. The irradiation processing was aimed at ion beam mixing of the sandwich to obtain a nanoparticulate composite. This was done by $1.6 \times 10^{16} \mathrm{Au}^{2+}$ ions $/ \mathrm{cm}^{2}$ of $4.5 \mathrm{MeV}$ energy having an ion range of nearly $1 \mu \mathrm{m}$, far beyond the sandwich dimensions [11].

Ion implantation was applied to silica glass (HERASIL grade) using $1 \times 10^{17} \mathrm{Ag}^{+}$ions $/ \mathrm{cm}^{2}$ of $150 \mathrm{keV}$ energy [11]. During implantation the sample holder was cooled with water to prevent target heating. This irradiation causes implantation of $\mathrm{Ag}^{+}$ions and precipitation of $\mathrm{Ag}$ particles in about $76 \mathrm{~nm}$ beneath the glass surface where a particulate layer of about $20 \mathrm{~nm}$ width is formed. TEM inspection of plane-view and cross-section preparation of all samples was done by means of a JEM 1010 operating at $100 \mathrm{kV}$ and a JEM 4010 operating at $400 \mathrm{kV}$.

\section{RESULTS AND DISCUSSION}

Electron irradiation causes liberation of electrons and holes as well as radiation damage in the glass matrix $[13,17]$. The defects due to irradiation are induced by bond cleavage of bridging oxygen [17] or oxygen displacement at higher energies [18]. The presence of non-bridging oxygen hole centres in electron irradiated samples was confirmed by electron paramagnetic resonance measurements [16]. Because of the strong electron trapping ability of $\mathrm{Ag}^{+}, \mathrm{Ag}^{0}$ is readily formed and mobile defects in the irradiated area enable precipitation of nanoparticles at room temperature. The largely enhanced diffusion results in a high concentration $(3.5 \%)$ of mostly single crystalline Ag nanoparticles of nearly spherical shape. A typical TEM micrograph is shown in Figure 1. These Ag nanoparticles exhibit rather small size of $4.2 \pm 0.9 \mathrm{~nm}$ and narrow size distribution [12]. The $\mathrm{Ag}^{+} \rightarrow \mathrm{Ag}^{0}$ conversion is much higher ( $\sim 4 \%$ ) and the nanoparticle formation proceeds much faster as compared to the ion exchange plus thermal processing route [6], but the particle arrangement is quite homogeneous. 


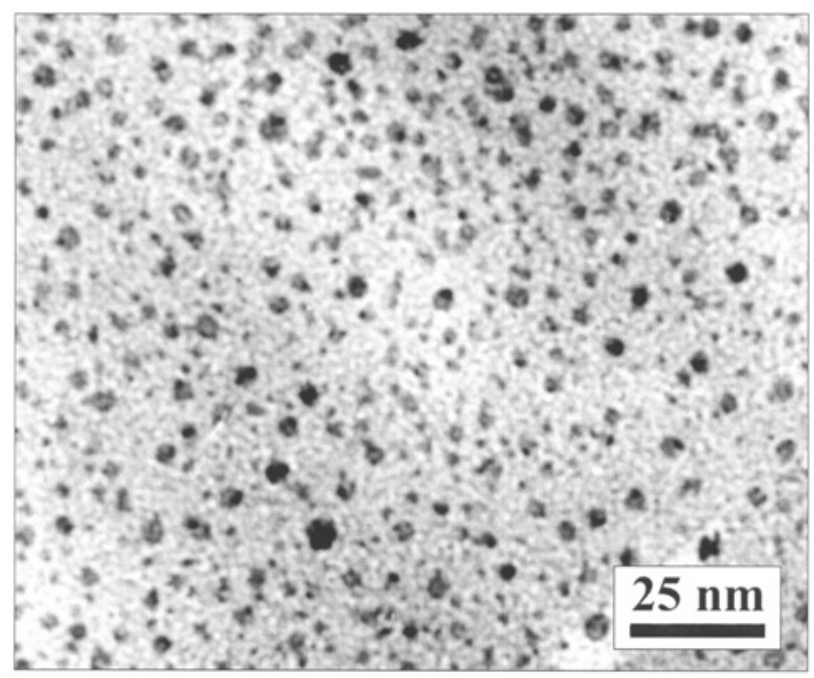

FIGURE 1 Ag nanoparticles formed in ion-exchanged glass upon exposure to $100 \mathrm{keV}$ electron irradiation.

High-energy laser pulse resonant excitation of metal particle surface plasmons results in size reduction and formation of very small particles, surrounding the primary particles like a halo. This specific configuration is shown in Figure 2(a). The particle modification is assumed to start upon intense and short enough laser pulses by ejection of electrons and $\mathrm{Ag}^{+}$ion desorption. Irreversible changes of the particle shape occur when using linear polarised pulses. Initially spherical particles are deformed and uniformly arranged with their long axes perpendicular to the polarisation direction [15]. This deformation is probably caused by the strong electrostatic field built up along the polarisation direction [19]. Because of high supersaturation in the matrix, electrons and $\mathrm{Ag}^{+}$ions recombine and precipitate in a certain distance of the primary particles. Mass balance considerations based on measurement of the halo particle size in comparison to the primary particle size reveal that the latter are diminished accordingly. Occasionally, particles with spherical cavities are found whose

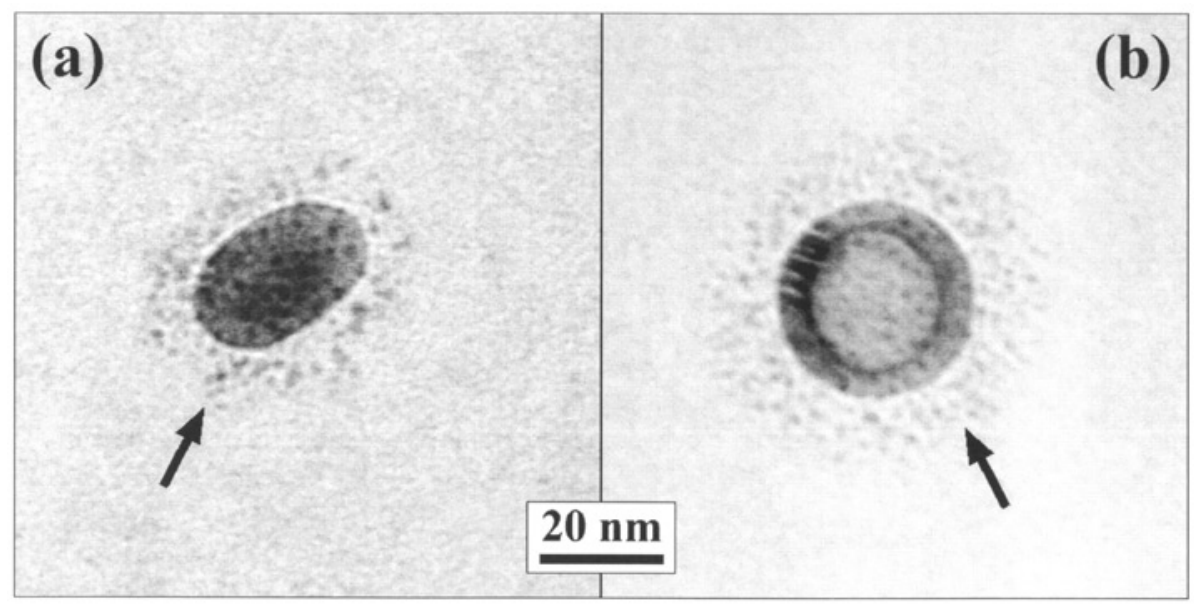

FIGURE 2 Ag nanoparticles in glass modified by polarised laser pulses: (a) deformation, (b) cavity. Arrows point to halo particles. 
volume amounts to that of the corresponding halo nanoparticles. These peculiar structures, an example is shown in Figure 2(b), most probably result from heating above the melting point and expelling some material, followed by very rapid solidification starting at the inner surface of the spherical hollow left in the matrix. The outside-in direction is usually observed in melting/solidification studies of embedded metal nanoparticles [20].

Ion irradiation causes competition of homogeneous mixing and lateral segregation of the metal layer sandwiched in between silica films by recoil implantation and enhanced diffusion after the collision cascade [11,12,21]. Minimisation of the interface energy leads to balling into uniformly arranged particles of spheroidal shape. As may be recognised from the crosssection micrograph shown in Figure 3 the long axes of these spheroidal particles of about 1.7 mean aspect ratio are uniformly arranged parallel to the irradiation direction. The micrograph also shows that in addition to the primary particles of about 5 to $50 \mathrm{~nm}$ size $\left(d_{\text {mean }}=\right.$ $18.9 \pm 8.5 \mathrm{~nm}$ ) smaller secondary particles of about $2.8 \mathrm{~nm}$ size occur. These secondary particles are found at two types of location. They decorate the previous position of the metal layer (see open arrow), and they surround the primary particles like a halo (see solid arrow). The first can be understood as remainings of lateral segregation which resisted further displacement because of their small size. The halo particles, on the other hand, are arranged in a certain distance from the primary particles, seen in plane-view as forming rings around them. The appearance of halo particles is more distinct on the side opposite to the incident ion beam, reflecting the directional nature of recoil implantation.

$\mathrm{Ag}^{+}$ion implantation into silica glass under the above mentioned conditions produces a high supersaturation of metal in the host and favours rapid precipitation of nanoparticles at room temperature. According to the ion energy applied, particle formation occurs in a rather thin layer just beneath the surface with non-uniform depth profile $[8,11]$. Arrangement and size of Ag nanoparticles obtained at low doses $\left(10^{15}\right.$ to $\left.10^{16} \mathrm{ions} / \mathrm{cm}^{2}\right)$ are similar to the electron irradiated sample. With increasing dose their size distribution width and depth distribution inhomogeneity distinctly enlarge. Upon implanting $1 \times 10^{17}$ ions $/ \mathrm{cm}^{2}$, nanoparticles of about 1.5 to $40 \mathrm{~nm}$ size $\left(d_{\text {mean }}=9.6 \pm 6.8 \mathrm{~nm}\right)$ are formed where a considerable part is above $20 \mathrm{~nm}$. Thus, the total fraction of silver incorporated in the implanted layer reaches a considerable amount as does the probability that ions hit already existing nanoparticles. Ion impact, due to the increased ion stopping cross-section

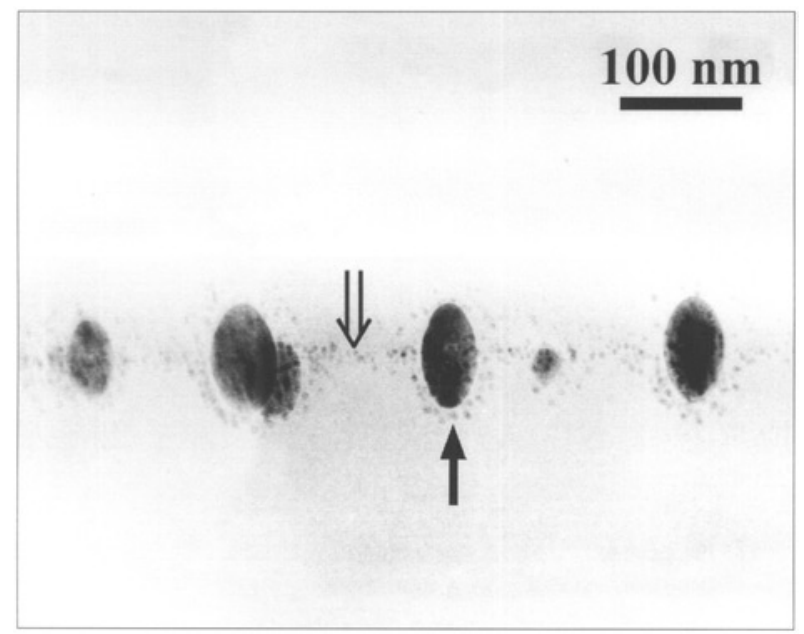

FIGURE 3 Ag nanoparticles by ion beam mixing $\left(4.5 \mathrm{MeV} \mathrm{Au}^{+}\right)$of a silica/Ag/silica sandwich. 


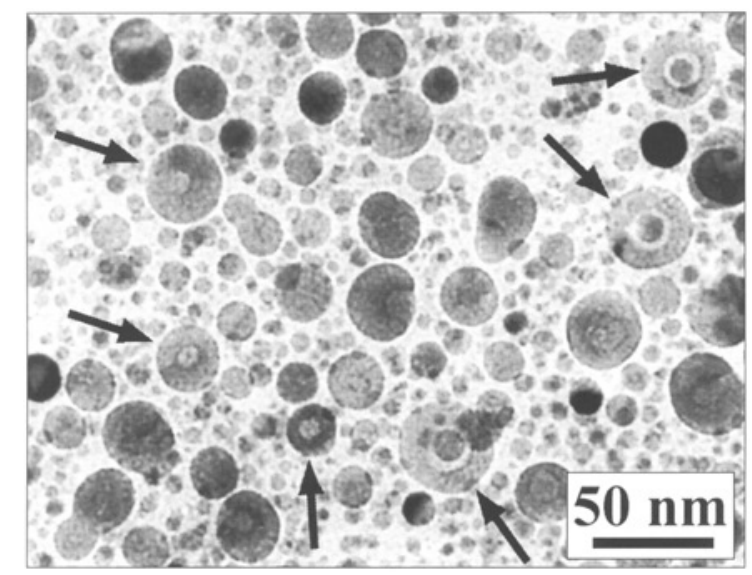

FIGURE $4 \mathrm{Ag}$ nanoparticles by implantation of $1 \times 10^{17} \mathrm{Ag}^{+}$ions of $150 \mathrm{keV}$ into silica glass. Arrows point to circular and ring-like cavities.

of large particles, leads to local melting, mass redistribution and the formation of cavities which partly have a complex appearance as shown in Figure 4. The formation of voids and hollow particles was reported up to now only for precipitates formed by ion implantation plus thermal processing [22]. The formation of craters in solid surfaces by ion beam irradiation giving rise to ring-like structures was reported for a broad range of energies [23]. Ioninduced cratering because of thermal spike effects in thin films of Au created features with concentrically arranged material of circular shape from which a ring-like appearance results [24], comparable to configuration and dimensions of those shown in Figure 4. Although crater formation may be impeded by about $50 \mathrm{~nm}$ silica the ions must pass through before impacting Ag particles, the above interpretation appears quite reasonably.

\section{CONCLUSIONS}

Various methods of irradiation-assisted processing have been utilised to induce, promote or modify formation of metal nanoparticles in silicate glass without thermal treatment. While electron beam irradiation applied to Ag doped glass creates defects in the matrix being rather effective in forming particles of uniform size, shape and arrangement, high-energy irradiation of the glass by very high ion fluences or ultrashort laser pulses results in quite inhomogeneous particle configurations of unique type. These are (i) spheroidal shape modifications with uniform arrangement of the long axes along certain directions of laser polarisation or ion movement, (ii) formation of very small secondary particles arranged like a halo around the primary ones, and (iii) creation of cavities and craters in particles of large interaction cross-section. The structures resulting from such spike effects should have only limited thermal stability, which will be the subject of future work.

\section{Acknowledgements}

The authors thank K.-J. Berg for preparation of Ag nanoparticle containing glass samples used in laser irradiation experiments. This work was partly supported by SFB 418 of the Deutsche Forschungsgemeinschaft. 


\section{References}

[1] Chakraborty, P. (1998). J. Materials Science, 33, 2235.

[2] Gonella, F. and Mazzoldi, P. (2000). Handbook of Nanostructured Materials and Nanotechnology, Vol. 4. Academic Press, San Diego, p. 81.

[3] Kreibig, U., Gartz, M., Hilger, A. and Hövel, H. (1998). Adv. Met. Semicond. Clusters, Vol. 4. JAI Press, Stanford, p. 345.

[4] Hofmeister, H., Drost, W.-G. and Berger, A. (1999). NanoStructured Materials, 12, 207.

[5] Ramaswamy, R. V. (1988). J. Lightwave Technol., 6, 984.

[6] Dubiel, M., Hofmeister, H. and Schurig, E. (1998). Recent Res. Devel. Appl. Phys., 1, 69.

[7] Stepanov, A. L., Hole, O. E. and Townsend, P. D. (1999). J. Non-Crystalline Solids, $244,275$.

[8] Dubiel, M., Hofmeister, H., Schurig, E., Wendler, E. and Wesch, W. (2000). Nucl. Inst \& Meth. B, 166-167, 871.

[9] Pivin, J. C., Sendova-Vassileva, M., Nikolaeva, M., Dimova-Malinovska, D. and Martucci, A. (2002). Appl. Phys. A, 75, 401.

[10] Pivin, J. C. (2000). Materials Sci. Engn. A, 293, 30.

[11] Pivin, J. C., Garcia, M. A., Llopis, J. and Hofmeister, H. (2002). Nucl. Instr. \& Meth. B, 191, 794.

[12] Hofmeister, H., Thiel, S., Dubiel, M. and Schurig, F. (1997). Appl. Phys. Lett., 70, 1694.

[13] Chen, S., Akai, T., Kadono, K. and Yazawa, T. (2001). Appl. Phys. Lett., 79, 3687.

[14] Rainer, T. (2000). Verh. Deutsche Physikal. Ges., 4, 416.

[15] Kaempfe, M., Seifert, G., Berg, K.-J., Hofmeister, H. and Graener, H. (2001). Eur. Phys. J. D, 16, 237.

[16] Thiel, S., (1995). PhD thesis, Martin Luther University Halle, p. 54.

[17] Stevens-Kalceff, M. A. (2000). Phys. Rev. Lett., 84, 3137.

[18] Goldberg, M., Fitting, H. J. and Trukhin, A. (1997). J. Non-Crystalline Solids, 220, 69.

[19] Kaempfe, M. (2000). PhD thesis, Martin Luther University Halle, p. 75.

[20] Kofman, R., Cheyssac, P. S. and Aouaj, A. (1994). Surf Sci., 303, 231.

[21] Pivin, J. C. and Rizza, G. (2000). Nucl. Instr. \& Meth. B, 166-167, 115.

[22] Meldrum, A., Honda, S., White, C. W., Zuhr, R. A. and Boatner, L. A. (2001). J. Mater. Res., 16, 2670.

[23] Bringa, E. M., Nordlund, K. and Keinonen, J. (2001). Phys. Rev. B, 64, 235426-1.

[24] Donnelly, S. E. and Birtcher, R. C. (1999). Phil. Mag. A, 79, 133. 